\title{
Millimeter-wave enabled PAM-4 data transmission over hybrid FSO-MMPOF link for access networks
}

\author{
Saeed Iqbal ${ }^{1} \cdot$ Aadil Raza $^{2} \cdot$ Muhammad Fasih Uddin Butt $^{1,3}$ (1) $\cdot$ Jawad Mirza $^{4} \cdot$ Muhammad Iqbal $^{5} \cdot$ \\ Salman Ghafoor ${ }^{6} \cdot$ Mohammed El-Hajjar $^{3}$
}

Received: 20 November 2020 / Accepted: 31 March 2021 / Published online: 25 April 2021

(c) The Author(s) 2021

\begin{abstract}
In this paper, we propose a full duplex architecture based on a hybrid link composed of free space optics (FSO) and multimode plastic optical fiber (MMPOF) for short-range wireless access networks. The proposed architecture employs mode group division multiplexing (MGDM) and wavelength reuse techniques to transmit data between central unit (CU) and radio access units (RAUs). An optical frequency comb source to generate multiple optical sidebands is realized using a single laser source to provide $60 \mathrm{GHz}$ millimeter-wave (mm-wave) signals at each RAU by simultaneously transmitting PAM-4 signal on linearly polarized (LP) modes of each optical sideband. Data rate of $2 \times 12 \mathrm{Gbps}$ at mm-wave frequency of $60 \mathrm{GHz}$ is achieved for both downlink (DL) and uplink (UL) transmissions. The transmission of $2 \times 12$ Gbps PAM-4 signal over hybrid FSO-MMPOF link is investigated at different values of refractive index structure parameter $\left(C_{n}^{2}\right)$ by employing $\log$ normal (LN) FSO channel model to support three RAUs in a ring topology. The acceptable receiver sensitivities below -5 and $-0.6 \mathrm{dBm}$ for DL and UL transmissions are achieved, respectively. The proposed hybrid architecture can be a potential candidate for future communication networks.
\end{abstract}

Keywords Multimode plastic optical fiber $\cdot$ Free space optics $\cdot$ Mode group division multiplexing $\cdot$ Millimeter wave $\cdot$ Pulse amplitude modulation

Muhammad Fasih Uddin Butt

fasih@comsats.edu.pk; M.F.U.Butt@ soton.ac.uk

Saeed Iqbal

saeed@biit.edu.pk

1 Department of Electrical and Computer Engineering, COMSATS University Islamabad, Islamabad, Pakistan

2 Department of Physics, COMSATS University Islamabad, Islamabad, Pakistan

3 School of Electronics and Computer Science, University of Southampton, Southampton, UK

4 SEECS, Photonics Research Group, Islamabad, Pakistan

5 Department of Computer and Information Sciences, Pakistan Institute of Engineering and Applied Sciences (PIEAS), Islamabad, Pakistan

6 SEECS, National University of Sciences and Technology, Islamabad, Pakistan

\section{Introduction}

Among the requirements of future communication networks is to provide higher data rate, improved performance, as well as lower latency and ubiquitous services to the end users. Hence, to achieve these requirements, it is advantageous to exploit simultaneously both optical and wireless communication techniques. Optical fiber communication, despite having higher bandwidth and capacity, is not suitable for seamless coverage and mobility [1,2]. On the contrary, wireless communication provides better coverage and mobility, despite having limited radio frequency (RF) spectrum bandwidth resources and several sources of impairments [3]. To meet the unabated data rate demands, future communication networks can be an integration of optical and wireless communication technologies, which is referred to as radio over fiber (RoF) technology [4-6].

Furthermore, for the distribution of multiple wireless services for ultra-high bandwidth applications such as 3D and $4 \mathrm{~K}$ videos in future communication networks, the fiber to the X (FTTX) technology has been adopted worldwide [7]. 
Here, X in FTTX represents destinations, such as homes, buildings, and premises, and can therefore be termed as fiber to the home (FTTH), fiber to the building (FTTB), and fiber to the premises (FTTP), respectively. It has been reported in [7] that approximately 500 million FTTX connections have been deployed across the world till 2020 and this number is expected to grow further in the future. However, the deployment of FTTX networks in a congested residential or commercial areas involve large deployment cost of fiber. Generally, network providers opt for wireless signal transmission where they just need to install base station at different locations instead of digging the ground for laying fiber. Therefore, a potential solution would be free space optics (FSO)-based solutions for backhaul high data rate transmission. FSO can provide increased capacity, immunity to electromagnetic interference, and seamless transmission of high data rate optical signals at reduced installation and maintenance costs [8-11]. A hybrid link based on FSO and multimode plastic optical fiber (MMPOF) can be a potential solution for next-generation wireless access networks, because installation of FSO backhaul link is suitable in geographical areas such as hilly areas and canals, etc., where fiber cannot be deployed. MMPOF is more suitable for fronthaul links in indoor environments due to its larger core size as compared to single-mode fiber (SMF). SMF has advantages over MMPOF in terms of bandwidth, dispersion, and attenuation, but the installation cost of SMF and single-mode transceivers is higher than MMPOF due to a need of trained people and high precision devices. In addition, MMPOF as compared to multimode glass optical fiber has smaller bending radius which makes its installation comparatively easy [12, 13]. In the literature, a hybrid link-based architecture using $11 \mathrm{~m} \mathrm{FSO}$ and $100 \mathrm{~m}$ MMPOF has been presented in [14] where the effects of fog and atmospheric turbulence for 11 $\mathrm{m}$ FSO link have been considered. In [15], the FSO link for different weather conditions has been investigated with passive optical network (PON) where different lengths of FSO channel with $25 \mathrm{~km}$ SMF has been achieved for $10 \mathrm{Gbps}$ on-off keying (OOK) data rate. Additionally, duplex link of $50 \mathrm{~km} \mathrm{SMF}$ and $100 \mathrm{~m}$ FSO has been reported in [16] to achieve $10 \mathrm{Gbps}$ data rate. Furthermore, an FSO-based FTTH network has been demonstrated by using $40 \mathrm{~km} \mathrm{SMF}$ and $100 \mathrm{~m}$ FSO link to support 20/10 Gbps at 50/20 GHz millimeter-wave (mm-wave) communication [17].

Therefore, employing FSO for backhaul and MMPOF for fronthaul can provide a better solution for rural and urban areas. MMPOF is flexible, cheap, and easy to handle, and hence, it is attractive for in-building networks [6]. Furthermore, mode group division multiplexing (MGDM) in MMPOF by exploiting LP01 and LP11 modes provides additional degrees of freedom to achieve optical multiple input and multiple output (MIMO) transmission, which ultimately increases the throughput and number of users of the network $[18,19]$. To support higher data rates in access network, mm-wave communication can be used for the wireless link between the user equipment (UE) and the radio access unit (RAU). Modulation schemes, such as quadrature amplitude modulation (QAM), quadrature phase shift keying (QPSK), and pulse amplitude modulation-N (PAM-N), etc., have been used to achieve high throughput for mm-wave enabled RoF systems [20]. However, these higher order modulation schemes increase the complexity and cost of the system. On the contrary, PAM-N is implemented by utilising intensity modulation/ direct detection (IM/DD), which is simple and cost efficient as compared to its counterparts. Lately, PAM-4 has been standardized by IEEE P802.3bs $400 \mathrm{GbE}$ task force owing to its lower power consumption, higher spectrum efficiency, and inexpensive implementation [21-23].

To fulfill the requirements of future communication networks, a hybrid link based on MMPOF and FSO has been presented as a viable solution. The limitation of electronic techniques for the generation of $\mathrm{mm}$-wave signals is solved by optical techniques such as heterodyne detection (HD). Additionally, the use of FSO link between central unit $(\mathrm{CU})$ and residential gateway $(\mathrm{RG})$ instead of the MMPOF further reduces the capital expenditure/ operational expenditure (CAPEX/OPEX) while introducing extra mobility. The main objective in this work is to study the effects of integration of MMPOF and FSO on the system performance. The channel impairments of MMPOF and FSO can affect the system performance in such a way that the acceptable bit error ratio (BER) at the targeted forward error correction (FEC) limit is difficult to achieve. To solve this issue, we have optimized the data rates, MMPOF length, FSO link range, and physical parameters of FSO transceiver in such a way that the BER at the targeted FEC limit is achieved successfully for both downlink (DL) and uplink (UL) transmissions. The system has enough robustness and resilience to the MMPOF and FSO impairments like, model crosstalk, atmospheric attenuation and turbulence, etc.

In this paper, we demonstrate all-optical generation and transmission of mm-wave signals over hybrid FSO-MMPOF link, where MMPOF is used to connect all RAUs in a ring architecture. Multiple optical carriers are generated using a dual-drive Mach-Zehnder modulator (DDMZM) and a continuous wave $(\mathrm{CW})$ laser source. Duplex transmission of $2 \times 12$ Gbps PAM- 4 data signals between the $\mathrm{CU}$ and the RAUs over hybrid FSO-MMPOF link is achieved exploiting MGDM. Additionally, all-optical techniques are used to generate mm-wave signals for DL at the RAUs; while for the UL transmission, the received mm-wave signals at the RAUs from UEs are transmitted to the $\mathrm{CU}$, after processing, using carrier reuse techniques. This simulation is performed in commercially available software: OptiSystem 17. 
Based on the above discussion, the novel contributions of our work are summarized as follows:

1. The performance of the designed duplex PAM-4-based optical transmission system is analyzed for a hybrid link of total length $600 \mathrm{~m}$. This design is capable to support $60 \mathrm{GHz}$ mm-wave services at throughput of $12 \mathrm{Gbps}$.

2. The FEC limit of BER at $3.8 \times 10^{-3}$ is achieved for PAM-4 received signal for different values of refractive index structure parameter $\left(C_{n}^{2}\right)$ of log-normal (LN) channel such as $5 \times 10^{-16}, 5 \times 10^{-15}$ and $5 \times 10^{-14} \mathrm{~m}^{-2 / 3}$ at lower receiver sensitivities.

3. We also show that an optical comb based on a single laser source facilitates duplex transmission in all three RAUs simultaneously in a ring topology fashion. LP modes enabled mm-wave services are achieved at each RAU.

The rest of the paper is organised as follows. In Sect. 2, the proposed architecture is discussed in detail, where the generation of multiple optical carriers, and the design and working of DL and UL transmission and receiver design for UE for $\mathrm{mm}$-wave are presented. Then, the performance analysis and results are discussed in Sect. 3, while in Sect. 4, conclusions of this work are presented.

\section{The proposed architecture}

The proposed system based on hybrid FSO-MMPOF link is shown in Fig. 1 where the RAUs are connected with the $\mathrm{RG}$ in a ring topology fashion using MMPOF, while the $\mathrm{RG}$ is connected to the $\mathrm{CU}$ using an FSO link. The CU is responsible for multi-wavelength generation, distribution, and data modulation, while each RAU performs photodetection and amplification followed by the $\mathrm{mm}$-wave signal transmission to the UE. At the CU, multi-wavelength signals are generated using DDMZM and RF source having frequency of $30 \mathrm{GHz}$, which will be discussed in subsect. 2.1. Six optical carriers $\lambda_{1}, \lambda_{2}, \lambda_{3}, \lambda_{4}, \lambda_{5}$, and $\lambda_{7}$ are separated using demultiplexer, as shown in Fig. 2 for both the DL and the UL transmission which support three RAUs that are connected to the $\mathrm{CU}$ in a ring topology, as shown in Fig. 1. We use $\lambda_{7}$ instead of $\lambda_{6}$ to ensure the frequency separation of $60 \mathrm{GHz}$ between $\lambda_{5}$ and $\lambda_{7}$ to generate $60 \mathrm{GHz}$ mm-wave signal at the RAU-3.

At the CU, optical MIMO is generated by passing each wavelength through mode generator to generate linearly polarized (LP) modes known as LP01 mode and LP11 mode of each wavelength, as shown in Fig. 2. Data are modulated with $\lambda_{1}, \lambda_{2}$, and $\lambda_{5}$ using MZM for DL transmission toward RAU-1, RAU-2, and RAU-3, respectively, while $\lambda_{3}, \lambda_{4}$, and $\lambda_{7}$ are left as unmodulated carriers, which are used for UL transmission of RAU-1, RAU-2, and RAU-3, respectively. PAM-4 data are modulated with LP01 and LP11 mode of $\lambda_{1}$, $\lambda_{2}$, and $\lambda_{5}$, as shown in Fig. 2. In Fig. 2, the implementation detail for DL transmission to RAU-1 is shown, while the DL transmissions for RAU-2 and RAU-3 are performed in a similar manner for RAU-1. Then, the multiplexed optical signal is transmitted over FSO link, where the FSO link has been modelled using LN FSO channel model [24]. After FSO transmission, the received optical signal is transmitted over MMPOF having length of $300 \mathrm{~m}$. The simulation parameters of MMPOF are the same as the commercially available MMPOF having model number Giga-POF-50SRChromis Fiberoptics [6, 18]. The combined received optical signal is fed to a $1 \times 8$ spatial demultiplexer (DEMUX) to separate each carrier. Then, the mm-wave signals are generated by HD and transmitted to the UE. Furthermore, the mm-wave signals received at RAUs from the UE are converted to optical signals and transmitted towards $\mathrm{CU}$, which is discussed in subsect.n 2.2.

Fig. 1 Application scenario of the proposed architecture

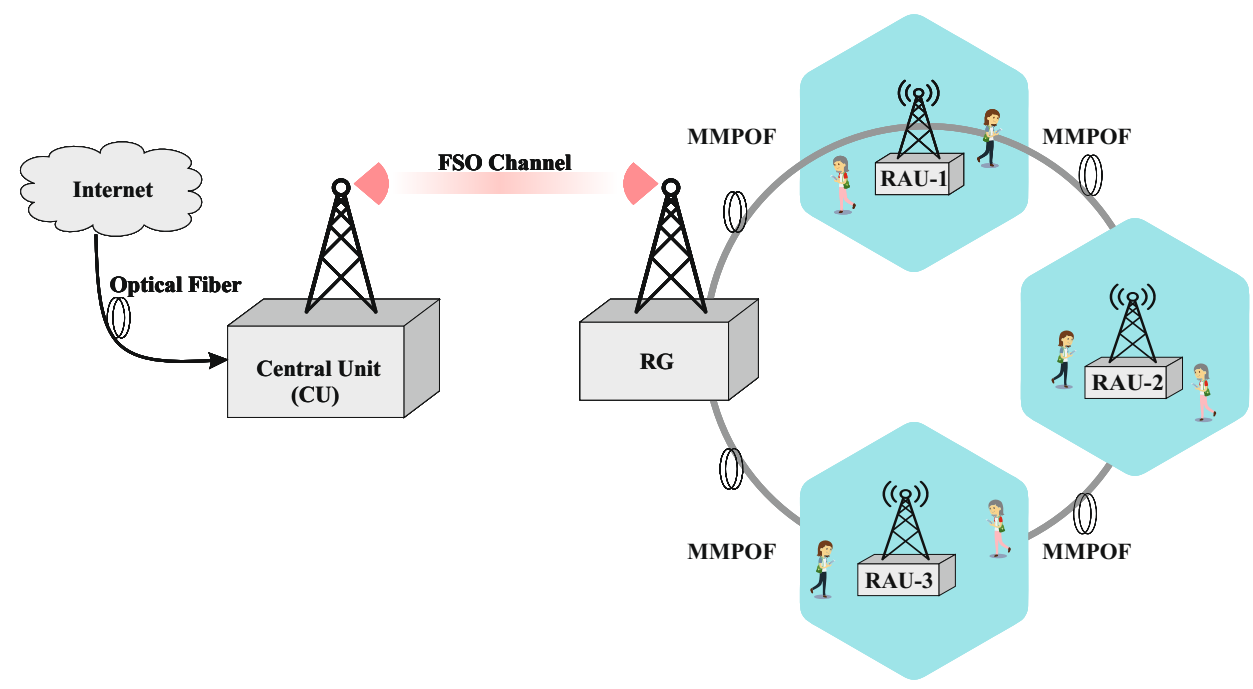




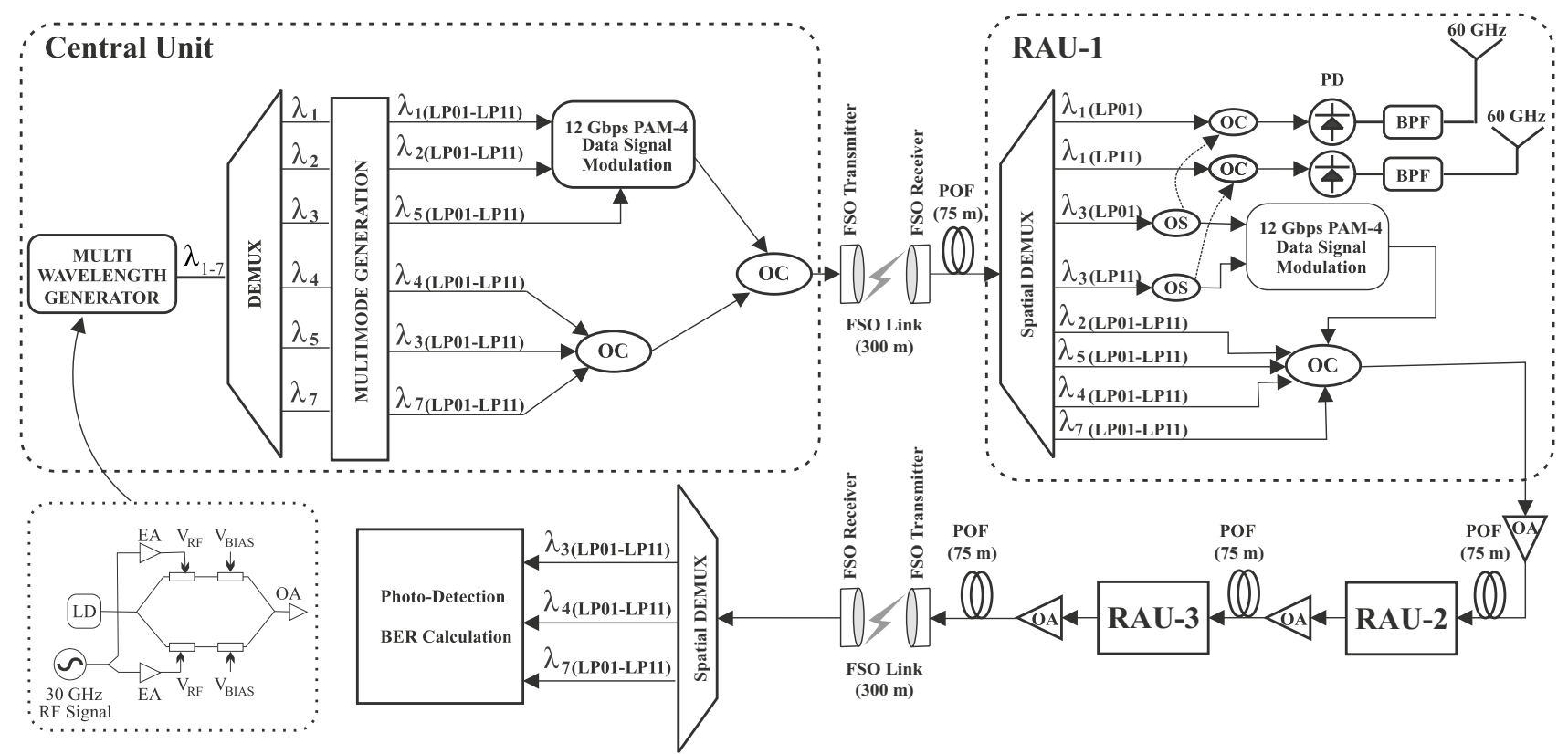

Fig. 2 The proposed architecture, $O S$ Optical splitter, $O A$ Optical amplifier, $O C$ Optical coupler, $F S O$ Free space optics, $B P F$ Band-pass filter, $P D$ Photodetector

\subsection{Downlink transmission}

The proposed model provides mm-wave services at high data rate to the end users, as shown in Fig. 1. To facilitate multiple RAUs, multiple optical carriers are required at the $\mathrm{CU}$ to transport the data. Multiple laser sources can be used to achieve the required number of carriers. However, this increases the cost of the overall communication system. One of the possible solutions is to generate multiple optical carriers from a single laser source by exploiting the MZM properties which generate multiple sidebands when modulated by an RF signal [25]. This can be achieved by modulating an RF signal with optical carrier of a single laser source, having peak wavelength of $1300 \mathrm{~nm}$, using a DDMZM, as shown in Fig. 2.

It can be seen in Fig. 2 that a laser diode (LD), an RF source of $30 \mathrm{GHz}$, and a DDMZM are used to generate multiple side bands. The chirping effect is the inherit property of Mach-Zehnder modulator (MZM) which is exploited for generation of multiple optical carriers. Chirping effect is defined as the change in optical carrier's phase at the output of the phase modulator (PM). The applied voltage to the branches of the DDMZM is controlled by electrical signal to produce the desire chirp which tends to change phase of optical signal passing through DDMZM [26]. This results in the generation of the multiple optical coherent carriers at the output of the DDMZM. The DDMZM is fed by the optical field of the LD which can be written as
$\zeta_{\mathrm{i}}(t)=\sqrt{P_{\mathrm{in}}} \exp \left(j 2 \pi f_{\mathrm{o}} t\right)$

where $f_{o}$ is frequency and $P_{i n}$ is the power of the optical carrier. The optical field at the output of the DDMZM is written as $[27,28]$

$\zeta_{\mathrm{o}}(t)=\frac{\zeta_{\mathrm{i}}(t)}{2}\left[\exp \left\{j \frac{(1+\delta) \pi v_{1}(t)}{v_{\pi}}\right\}+\exp \left\{j \frac{(1-\delta) \pi v_{2}(t)}{v_{\pi}}\right\}\right]$

where $v_{1}(t)$ and $v_{2}(t)$ are applied voltages to the upper and lower arms of the DDMZM, respectively. The $180^{\circ}$ change in phase is induced by $v_{\pi}$. $\delta$ is the chirp factor. The RF signals' applied voltage to DDMZM are selected as $v_{2}(t)=-v_{1}(t)$ to operate DDMZM in push-pull mode which is required to generate multiple side band of optical carrier. The RF signal applied to DDMZM is written as

$v_{1}(t)=\frac{1}{2}\left\{A_{\mathrm{rf}} \sin \left(\omega_{\mathrm{rf}} t\right)+A_{\mathrm{DC}}\right\}$

here, $A_{r f}$ and $A_{D C}$ are the amplitude of the RF and DC bias voltage, respectively, and $\omega_{r f}$ is the RF signal's angular frequency. By putting Eq. (3) in Eq. (2), we get

$$
\begin{aligned}
\zeta_{\mathrm{o}}(t)= & \frac{\zeta_{\mathrm{i}}(t)}{2}\left[\exp \left\{j \frac{(1+\delta) \pi\left(A_{\mathrm{rf}} \sin \left(\omega_{\mathrm{rf}} t\right)+A_{\mathrm{DC}}\right)}{2 v_{\pi}}\right\}\right. \\
& \left.+\exp \left\{-j \frac{(1-\delta) \pi\left(A_{\mathrm{rf}} \sin \left(\omega_{\mathrm{rf}} t\right)+A_{\mathrm{DC}}\right)}{2 v_{\pi}}\right\}\right] .
\end{aligned}
$$




$$
\begin{aligned}
\zeta_{\mathrm{o}}(t)= & \frac{\zeta_{\mathrm{i}}(t)}{2}\left[\exp \left\{j \kappa_{2} \sin \left(\omega_{\mathrm{rf}} t\right)\right\} \exp \left(j \kappa_{1}\right)\right. \\
& \left.+\exp \left\{-j \kappa_{4} \sin \left(\omega_{\mathrm{rf}} t\right)\right\} \exp \left(-j \kappa_{3}\right)\right],
\end{aligned}
$$

where

$$
\begin{gathered}
\kappa_{1}=\frac{\pi(1+\delta)}{2 v_{\pi}} A_{\mathrm{DC}}, \\
\kappa_{2}=\frac{\pi(1+\delta)}{2 v_{\pi}} A_{\mathrm{rf}}, \\
\kappa_{3}=\frac{\pi(1-\delta)}{2 v_{\pi}} A_{\mathrm{DC}}, \\
\kappa_{4}=\frac{\pi(1-\delta)}{2 v_{\pi}} A_{\mathrm{rf}} .
\end{gathered}
$$

The operating point of the DDMZM is set at quadrature point, so that intensity modulation is achieved. This mode of operation causes no distortion in the complete swing of the $\mathrm{RF}$ signal. Therefore, DC bias voltage is set as $v_{\pi} / 2$, peakto-peak modulation voltage is set equal to $v_{\pi}$ and the peak voltage of RF signal $A_{r f}$ is set equal to $v_{\pi} / 2$. Therefore, by putting $A_{r f}=A_{D C}=v_{\pi} / 2$ in Eq. (6), we get $\kappa_{1}=\kappa_{2}$ and $\kappa_{3}=$ $\kappa_{4}=\pi / 2-\kappa_{1}$. After substituting $\kappa_{2}, \kappa_{3}$ and $\kappa_{4}$ in terms of $\kappa_{1}$ in Eq. (5), the following equation is obtained:

$$
\begin{aligned}
\zeta_{\mathrm{o}}(t) & =\frac{\zeta_{\mathrm{i}}(t)}{2} \exp \left(j \kappa_{1}\right)\left[\exp \left\{j \kappa_{1} \sin \left(\omega_{\mathrm{rf}} t\right)\right\}\right. \\
& \left.-j \exp \left\{\left(-j\left(\frac{\pi}{2}-\kappa_{1}\right) \sin \left(\omega_{\mathrm{rf}} t\right)\right)\right\}\right] .
\end{aligned}
$$

Finally, Eq. (7) can also be written as follows [29]:

$$
\begin{aligned}
\zeta_{\mathrm{o}}(t)= & \frac{\sqrt{P_{\mathrm{o}}} \exp \left(j \kappa_{1}\right)}{2}\left[\left\{J_{\mathrm{o}}\left(\kappa_{1}\right)-j J_{\mathrm{o}}\left(\kappa_{1}-\frac{\pi}{2}\right)\right\} \exp \left(j 2 \pi f_{\mathrm{o}} t\right)\right. \\
& +\sum_{n=-\infty}^{\infty} J_{\mathrm{n}}\left(\kappa_{1}\right) \exp \left(j 2 \pi\left(f_{\mathrm{o}}+n f_{\mathrm{rf}}\right) t\right) \\
& \left.-j \sum_{n=-\infty}^{\infty} J_{\mathrm{n}}\left(\kappa_{1}-\frac{\pi}{2}\right) \exp \left(j 2 \pi\left(f_{\mathrm{o}}+n f_{\mathrm{rf}}\right) t\right)\right]
\end{aligned}
$$

In above expression, $J_{o}\left(\kappa_{1}\right)$ and $J_{n}\left(\kappa_{1}\right)$ are the Bessel functions of frequency $\left(f_{o}\right)$ and $n^{\text {th }}$ order, respectively. Here, $n$ is the generated number of the sidebands. The spacing between the sidebands is controlled by the RF signal's frequency $\left(f_{r f}\right)$, and hence, to keep a $30 \mathrm{GHz}$ frequency gap between sidebands, the frequency of the RF signal is chosen to be $30 \mathrm{GHz}$. Figure 3 shows multiple optical sidebands having sufficient power at the output of DDMZM which are generated by adjusting the biasing voltage and the amplitude of the RF signal as detailed in $[6,18]$. The proper selection of the sidebands will enable the generation of $60 \mathrm{GHz} \mathrm{mm}$ wave signal at each RAU. The maximum power fluctuation among the sidebands is around $5 \mathrm{dBm}$, as shown in Fig. 3 . Hence, any sideband can be used for data transmission. However, optical carriers $\lambda_{1}, \lambda_{2}, \lambda_{3}, \lambda_{4}, \lambda_{5}$, and $\lambda_{7}$ are chosen for the proposed architecture as a design example, because all of these carriers have almost the same power, as shown in Fig. 3. Moreover, to perform HD at the RAUs to achieve $\mathrm{mm}$-wave $(60 \mathrm{GHz})$ transmission, the selection of optical
Fig. 3 Spectrum of generated multiple optical sidebands

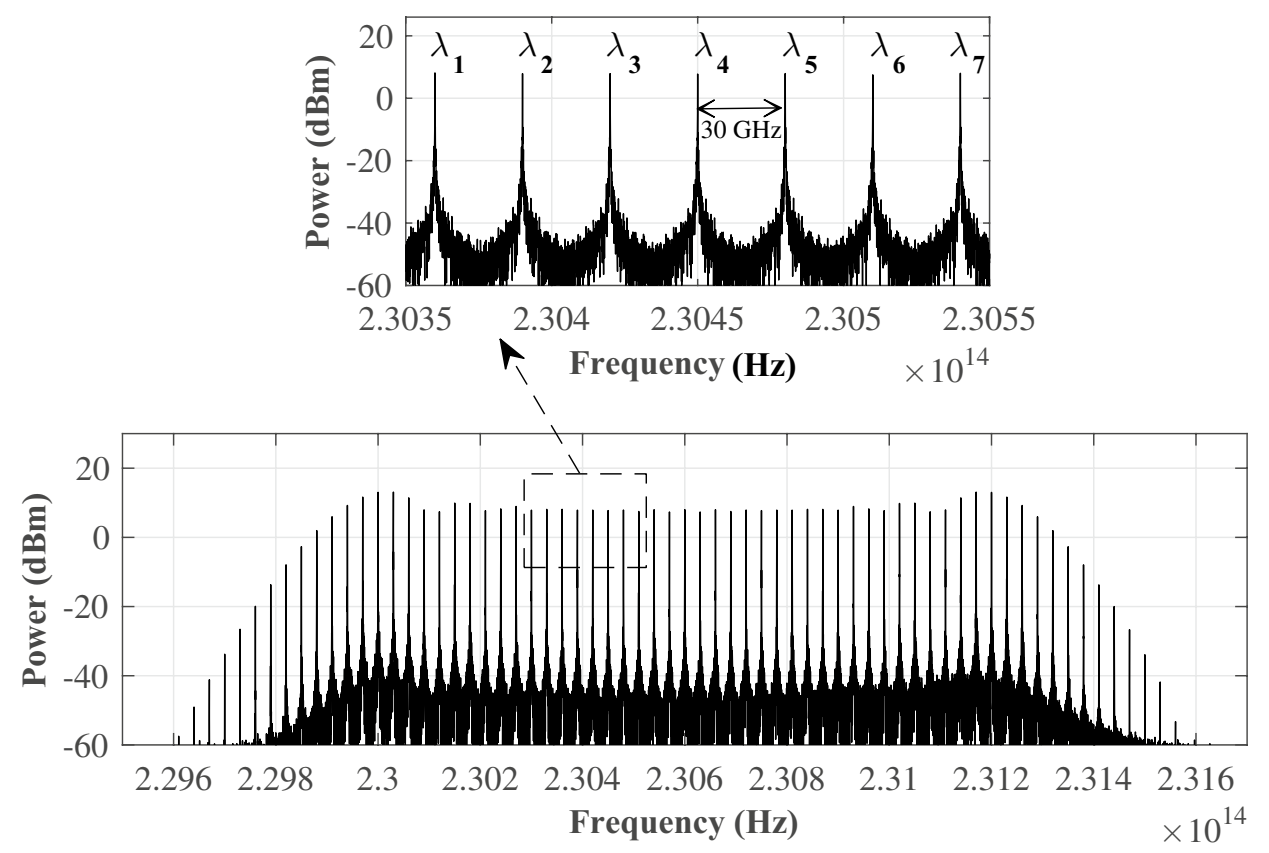


carriers for DL and UL is made on the basis that there must be frequency difference of $60 \mathrm{GHz}$ among them. Therefore, $\lambda_{1}, \lambda_{2}$, and $\lambda_{5}$ are used for DL and $\lambda_{3}, \lambda_{4}$, and $\lambda_{7}$ are utilised for UL, as can be observed from Fig. 2.

The signal at the output of DDMZM as shown in Fig. 2 is fed into a $1 \times 6$ WDM-DEMUX to separate the six optical carriers having high optical signal-to-noise ratio (OSNR) which are at wavelengths $\lambda_{1}, \lambda_{2}, \lambda_{3}, \lambda_{4}, \lambda_{5}$, and $\lambda_{7}$, as shown in Fig. 3. It may be noted that only these six wavelengths are selected specifically in the proposed system which are used both for DL and UL transmission. LP01 and LP11 modes of each wavelength are generated using mode generator [18], as shown in Fig. 2. For DL transmission, LP01 and LP11 modes of $\lambda_{1}, \lambda_{2}$, and $\lambda_{5}$, as shown in Fig. 2, are intensity modulated with 12 Gbps PAM- 4 signal using MZM. However, $\lambda_{3}, \lambda_{4}$, and $\lambda_{7}$ are left unmodulated to be used at the RAU for heterodyning and UL transmission and will be discussed in more detail in the next section. The modulated wavelengths $\left(\lambda_{1}, \lambda_{2}, \lambda_{5}\right)$ and unmodulated wavelengths $\left(\lambda_{3}, \lambda_{4}, \lambda_{7}\right)$ are multiplexed using WDM-MUX, as shown in Fig. 2. The multiplexed signal at the output of WDMMUX is then transmitted over FSO link with the help of FSO transmitter towards the RG, as shown in Fig. 1. The transmitted signal undergoes various impairments of the FSO channel which include turbulence induced fading, atmospheric attenuation, and polarization fluctuation effects $[24,30]$. The arbitrary fluctuation of the received intensity induced by the nonuniformities in atmospheric temperature and pressure is considered as prime contributor to signal degradation in the FSO link performance. Different channel models for FSO link have been reported in the literature [31]. The frequently employed FSO channel models are LN, $\mathrm{K}$, Gamma-Gamma, negative exponential, and Log normalRician models [31]. The LN channel model is used for weak turbulence in the case of clear weather conditions [32]. The variation in received signal intensity in LN channel model can be written by the following probability density function (PDF) [24, 33]:

$p_{I}(I)=\frac{1}{2 I \sqrt{2 \pi \sigma_{x}^{2}}} \exp \left[-\frac{\ln \left(I / I_{o}\right)^{2}}{8 \sigma_{x}^{2}}\right]$.

In the above expression, $I$ is the instantaneous light intensity, $I_{o}$ is the instantaneous light intensity without turbulence, and

$\sigma_{x}^{2}=0.307 C_{n}^{2} k^{7 / 6} L^{11 / 6}$.

Here, $\sigma_{x}^{2}$ is variance induced due to turbulence, $C_{n}^{2}$ is the refractive index structure parameter, $L$ is the length of the FSO link in $\mathrm{km}$, and $k=2 \pi / \lambda$ is the wave number. Typically, the value of $C_{n}^{2}$ varies from $10^{-17}$ to $10^{-12} \mathrm{~m}^{-2 / 3}$ for weak turbulence to strong turbulence, respectively [33]. After passing through hybrid LN-based FSO-MMPOF channel, the received signals at the RAUs are processed to generate $\mathrm{mm}$-wave signals which will then be transmitted to the UE. In the next section, the processing at RAUs is discussed in more details.

\subsection{Radio access unit}

The RAUs are connected in a ring topology, as shown in Fig. 1. The RAUs are responsible for generating mm-wave signals in the optical domain, and then, optical-to-electrical conversion is performed by using positive intrinsic negative (PIN) diode. After passing through a $75 \mathrm{~m}$ MMPOF, at RAU-1, a spatial DEMUX is used to separate each wavelength and LP01 and LP11 modes, as shown in Fig. 2. The design of mode filter embedded inside spatial DEMUX is realized by optimizing the interaction parameters of symmetric fiber couplers as demonstrated in [34]. The wavelength DEMUX used at each RAU has the following specifications. It has channel bandwidth of $0.75 \times$ symbol rate $=$ $0.75 \times 6 \mathrm{GBaud}=4.5 \mathrm{GHz}$. Since the frequency separation between optical sidebands is $30 \mathrm{GHz}$ and the channel bandwidth is $4.5 \mathrm{GHz}$, therefore, there is an ignorable crosstalk between the DEMUX channels.

To generate mm-wave signal, LP01 mode of $\lambda_{1}$ which is modulated by PAM-4 signal and the unmodulated LP01 mode of $\lambda_{3}$ are given as an input to the optical coupler (OC) where the spacing between these wavelengths is $60 \mathrm{GHz}$. The combined optical signal at the output of the $\mathrm{OC}$ is given by the following expression:

$r(t)=a I(t)\left[m(t) A_{2} \cos \left(2 \pi f_{1} t\right)+A_{3} \cos \left(2 \pi f_{3} t\right)\right]$.

Therefore, the intensity of the received optical signal can be written as

$r(t)=a I(t) m(t) A_{2} \cos \left(2 \pi f_{1} t\right)+A_{3} \cos \left(2 \pi f_{3} t\right)$.

After square law photo-detection, the output current of the photodetector (PD) can be expressed as [35]

$$
\begin{aligned}
i_{P D}(t)= & R[r(t)]^{2}, \\
i_{P D}(t)= & R\left[A_{2}^{2} a^{2} I^{2}(t) m^{2}(t)+A_{3}^{2}+A_{2}^{2} a^{2} I^{2}(t) m^{2}(t) \cos 2\left(\pi f_{1} t\right)\right. \\
& +A_{3}^{2} \cos 2 \pi f_{3} t+a I(t) m(t) A_{2} A_{3} \cos \left\{2 \pi\left(f_{1}-f_{3}\right) t\right\} \\
& \left.+a I(t) A_{2} A_{3} m(t) \cos \left\{2 \pi\left(f_{1}+f_{3}\right) t\right\}\right] .
\end{aligned}
$$

The double frequency terms $\left(f_{1}+f_{3}, 2 f_{1}\right.$, and $\left.2 f_{3}\right)$ are at RF frequencies and can be filtered using band-pass filter (BPF). Similarly, the DC terms are not transmitted by the antenna and are filtered before transmission. Hence, leaving only the mm-wave term of interest which is transmitted over the 
wireless channel, as shown in Fig. 2. Therefore, Eq. (14) reduces to

$i_{P D}(t)=\operatorname{RaI}(t) A_{2} A_{3} m(t) \cos \left\{2 \pi\left(f_{1}-f_{3}\right) t\right\}$.

The beating of wavelengths results in the generation of mmwave signal, which is obtained after passing the output of the PIN diode through BPF having center frequency at 60 $\mathrm{GHz}$ and bandwidth of $20 \mathrm{GHz}$. This BPF is used to pass only mm-wave signal at $60 \mathrm{GHz}$ frequency and stop all other noise like frequency components and then transmit to the UE.

At the UE, the received mm-wave signal is passed through a BPF to reject the out-of-band noise and then amplified using an electrical amplifier (EA). Self-mixing technique is used to down-convert the high-frequency electronic signal to baseband signal which is then filtered out using low-pass filter (LPF) to obtain the baseband data signal [6]. In digital signal processing, least mean squared (LMS) algorithm is employed to mitigate the channel impairments.

RAU-1 receives mm-wave signals from the UE which are then modulated with LP11 and LP01 of $\lambda_{3}$ and are transmitted towards RAU-2 and then RAU- 3 after coupling with other wavelengths $\lambda_{2}, \lambda_{4}, \lambda_{5}$, and $\lambda_{7}$ using MMPOF link, as shown in Fig. 2. The optical signal further travels through $75 \mathrm{~m}$ and $150 \mathrm{~m}$ over MMPOF for RAU-2 and RAU-3, respectively. The optical signals received at RAU-2 and RAU-3 are treated in a similar manner as in RAU-1. The received mm-wave signals at RAU-1, RAU-2, and RAU-3 from the UE are processed before transmission toward the $\mathrm{CU}$ at the respective RAU as discussed in the next section.

\subsection{Uplink transmission}

As shown in Fig. 1, each RAU is responsible to provide coverage in a specific area where mm-wave signals are received at the RAU from the UE, as shown in Fig. 1. At the RAU, these signals are processed before transmission to the CU. For UL transmission, $60 \mathrm{GHz}$ signal cannot be transmitted over MMPOF due to high losses at higher frequencies. Therefore, it may be noted that 12 Gbps PAM- 4 data signal is realized at the RAU for UL transmission by self mixing of the $60 \mathrm{GHz} \mathrm{mm}$-wave signal received from the UE. Then, a
LPF is used to filter out the PAM-4 signal which is shifted to baseband after self mixing. Then, LP01 and LP11 modes of $\lambda_{3}$ at RAU-1 are re-used and modulated by 12 Gbps PAM- 4 data signals using MZM, as shown in Fig. 2. Both of the modulated optical signals are then combined with $\lambda_{2}, \lambda_{4}$, $\lambda_{5}$, and $\lambda_{7}$ by WDM-MUX, and the combined optical signal at the output of WDM-MUX is transmitted towards RAU-2 and RAU-3 for UL transmission. Similarly, $\lambda_{4}$ and $\lambda_{7}$ are processed at RAU-2 and RAU-3, respectively, as $\lambda_{3}$ is processed at RAU-1. At RAU-3, finally, the modulated wavelengths $\lambda_{3}$, $\lambda_{4}$, and $\lambda_{7}$ are transmitted to the CU through hybrid FSOMMPOF link. The multiplexed optical signal obtained at the $\mathrm{CU}$ is fed into $1 \times 3 \mathrm{WDM}$-DEMUX to separate $\lambda_{3}, \lambda_{4}$, and $\lambda_{7}$, as shown in Fig. 2, and then, MF is used to separate LP01 and LP11 modes of each received wavelength. Each mode, LP01 and LP11, of $\lambda_{3}, \lambda_{4}$, and $\lambda_{7}$ are treated independently to receive data of RAU-1, RAU-2, and RAU-3, respectively. LP01 and LP11 modes of each wavelength modulated by PAM-4 signal are then sent to photo-detection.

\subsection{User equipment}

The received mm-wave signal is passed through a BPF for rejection of out-of-band noise and then amplified using an EA, as shown in Fig. 4. Self-mixing technique is used to down-convert the high-frequency electronic signal to baseband signal which is then filtered out using Gaussian LPF to obtain the baseband data signal [6]. In digital signal processing (DSP), least mean squared (LMS) algorithm is employed to mitigate the channel impairments. Eventually, BER is calculated to analyze the system performance.

\section{Performance analysis}

The performance of the proposed architecture is evaluated by analyzing the bit error ratio (BER). Table 1 summarizes major simulation parameters used in our simulations. BER analysis is performed on the received PAM-4 signals. DSP techniques are used for offline processing of received PAM-4 signals. After normalization, PAM-4 signal is re-sampled [36]. Then, the equalisation is performed with the aid of pilot signal using LMS algorithm for filter taps convergence.
Fig. 4 Design of UE receiver for mm-wave. $L P F$ Low-pass filter, $E S$ Electrical splitter, $E A$ Electrical amplifier, $B P F$ Bandpass filter

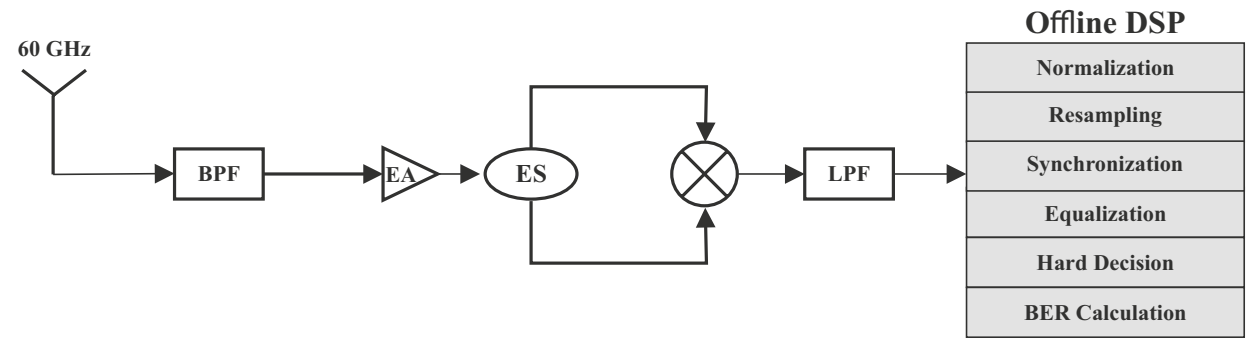


Table 1 Simulation parameters

\begin{tabular}{ll}
\hline Data rate of PAM-4 signal & $12 \mathrm{Gbps}$ \\
\hline Transmitter telescope diameter & $5 \mathrm{~cm}$ \\
Receiver telescope diameter & $20 \mathrm{~cm}$ \\
Beam divergence & $2 \mathrm{mrad}$ \\
Refractive index structure parameter & $5 \times\left(10^{-14}, 10^{-15}, 10^{-16}\right) \mathrm{m}^{-2 / 3}$ \\
Responsivity of PIN diodes & $0.8 \mathrm{~A} / \mathrm{W}$ \\
Optical amplifiers' gain & $20 \mathrm{~dB}$ \\
Optical amplifiers' noise figure & $4 \mathrm{~dB}$ \\
\hline
\end{tabular}

After the convergence, the decision directed mode is enabled. Finally, the hard decision is performed to calculate BER. The power of the received optical signal at the RAUs is varied using an optical attenuator to observe the effects on the BER. BER performance is analyzed on the basis of receiver sensitivity which is defined as the minimum received optical power (ROP) required to achieve BER of $3.8 \times 10^{-3}$. Figure $5 \mathrm{a}-\mathrm{c}$ shows the BER versus ROP curves for RAU-1, RAU-2, and RAU-3 for DL transmissions, while
Fig. 6a-c show the BER versus ROP curves for UL transmissions at different values of $C_{n}^{2}$. It may be observed from Fig. 5a that at $C_{n}^{2}=5 \times 10^{-16} \mathrm{~m}^{-2 / 3}$, the receiver sensitivity at FEC limit of BER of $3.8 \times 10^{-3}$ for DL PAM-4 signal received at RAU-1 is around $-12.5 \mathrm{dBm}$ for LP01 and $-10.8 \mathrm{dBm}$ for LP11 as compared with that of RAU-2 and RAU-3 which are around $-11.8 \mathrm{dBm}$ for LP01, $-9 \mathrm{dBm}$ for $\mathrm{LP} 11$, and $-10.2 \mathrm{dBm}$ for LP01, $-8.2 \mathrm{dBm}$ for LP11, respectively. It can be seen from Fig. 5a that the receiver sensitivity of RAU-1 is lower than RAU-2 and RAU-3.

The reason for the variation in the receiver sensitivities among DL PAM-4 transmission may be understood from the spectral plot of optical carriers, as shown in Fig. 3. It can observed from Fig. 3 that all wavelengths $\lambda_{1}, \lambda_{2}, \lambda_{3}$, $\lambda_{4}, \lambda_{5}$, and $\lambda_{7}$ have the same optical signal-to-noise ratio (OSNR). However, the reason for higher ROP of RAU-2 as compared to RAU-1 is due to the fact that the signal for RAU-2 has to travel longer distance than the signal for RAU-1 over MMPOF which results in higher degradation of the signal. The other reason may be polarization mode dispersion (PMD) and nonlinear effects of MMPOF. However, it is well known that PMD and nonlinear effects are
Fig. 5 BER versus received optical power for PAM-4 DL at a $\left(C_{n}^{2}=5 \times 10^{-16}\right)$, b $\left(C_{n}^{2}=5 \times 10^{-15}\right)$, and $\mathbf{c}$ $\left(C_{n}^{2} \stackrel{n}{=} 5 \times 10^{-14}\right)$

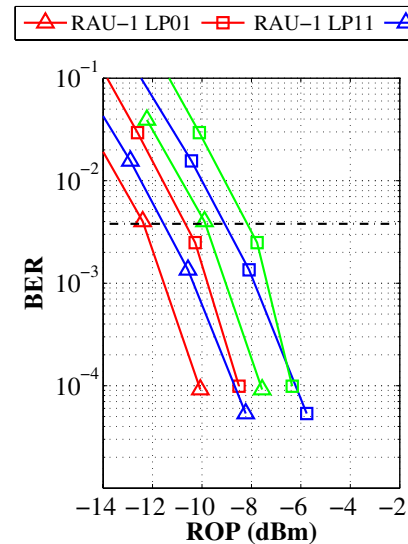

(a)

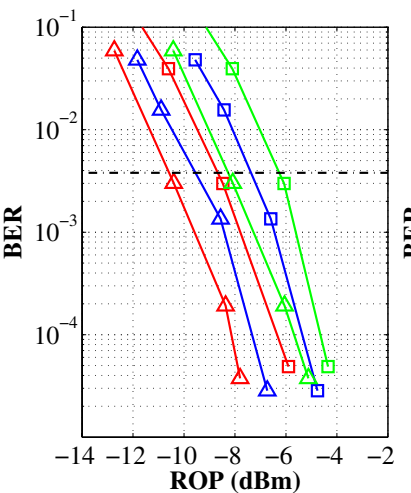

(b)

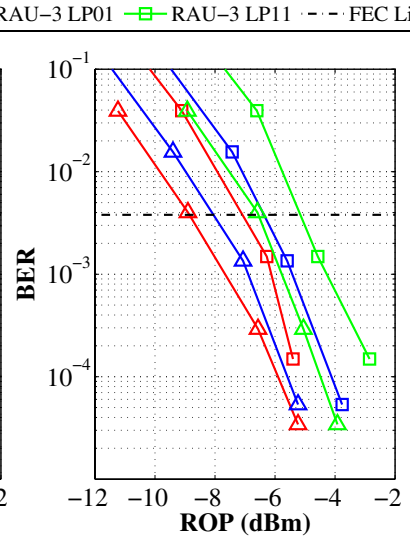

(c)
Fig. 6 BER versus received optical power for PAM-4 $\mathrm{UL}$ at $\mathbf{a}\left(C_{n}^{2}=5 \times 10^{-16}\right)$, b $\left(C_{n}^{2}=5 \times 10^{-15}\right)$, and c $\left(C_{n}^{2} \stackrel{n}{=} 5 \times 10^{-14}\right)$

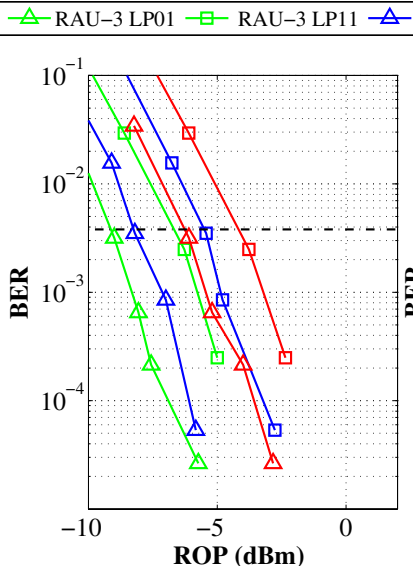

(a)

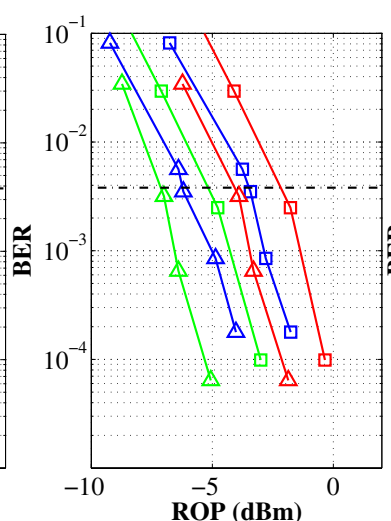

(b)

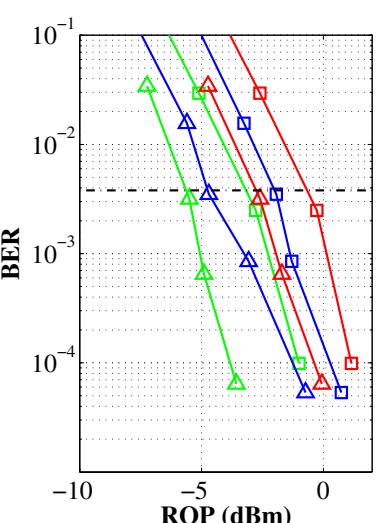

(c) 
limiting factors for long-haul optical transmission systems and at high transmitted laser's power, respectively [37, 38]. However, in our work, the power of the transmitted signal is lower for short-range communication; therefore, the aforementioned effects can be ignored. In addition, the large effective area of the MMPOF may also reduce the nonlinear effects and thus having no detrimental effects on signal quality. ROP at FEC limit of RAU-3 is higher, because $\lambda_{5}$ has to travel longer distance to reach RAU-3 as compared to $\lambda_{1}$ and $\lambda_{2}$. Similarly, UL BER curves of PAM- 4 data are shown in Fig. 6a. It can be seen from Fig. 6a that FEC limit at RAU-3 is achieved at $-9.1 \mathrm{dBm}$ for LP01 and $-6.9 \mathrm{dBm}$ for LP11. FEC limit for RAU-2 and RAU-1 is achieved at $-8.6 \mathrm{dBm}$ for LP01, $-5.2 \mathrm{dBm}$ for LP11 and $-6.6 \mathrm{dBm}$ for LP01, $-4.4 \mathrm{dBm}$ for LP11, respectively. It can be seen in Fig. 6a that the FEC limit of BER for RAU-3 is achieved at lower ROP than RAU-1 and RAU-2, because shorter distance over MMPOF is traveled by $\lambda_{7}$.

On increasing the value of $C_{n}^{2}$ parameter up to $5 \times 10^{-15} \mathrm{~m}^{-2 / 3}$, the minimum values of receiver sensitivities for DL PAM-4 signal received at RAU-3 are around $-10.5 \mathrm{dBm}$ for LP01 and $-8.3 \mathrm{dBm}$ for LP11, as shown in Fig. 5b. FEC limit at RAU-2 is around $-9.5 \mathrm{dBm}$ for LP01 and $-7.2 \mathrm{dBm}$ for LP11 and at RAU-1 is around $-8.2 \mathrm{dBm}$ for LP01 and $-6.3 \mathrm{dBm}$ for LP11. Likewise, the minimum receiver sensitivities for UL PAM-4 data at RAU-3 are $-7.2 \mathrm{dBm}$ for LP01 and $-5.2 \mathrm{dBm}$ for LP11, as presented in Fig. 6b. FEC limit for RAU-2 and RAU-1 is achieved at $-6.1 \mathrm{dBm}$ for LP01, $-3.8 \mathrm{dBm}$ for LP11 and $-3.8 \mathrm{dBm}$ for LP01, $-2.2 \mathrm{dBm}$ for LP11, respectively.

Figure $5 \mathrm{c}$ shows the DL received data's receiver sensitivities for $C_{n}^{2}=5 \times 10^{-14} \mathrm{~m}^{-2 / 3}$. At RAU-1, ROP $-9 \mathrm{dBm}$ for LP01 and $-7 \mathrm{dBm}$ for LP11 are achieved, while for RAU-2 and RAU-3, the values of ROPs are $-8.2 \mathrm{dBm}$ for $\mathrm{LP} 01,-6.8 \mathrm{dBm}$ for LP1 1 and $-6.9 \mathrm{dBm}$ for LP01, $-5 \mathrm{dBm}$ for LP11, respectively.

Figure $6 \mathrm{c}$ shows BER curves for UL transmission. It can observed from Fig. $6 \mathrm{c}$ that FEC limit at RAU-3 is achieved at $-5.8 \mathrm{dBm}$ for LP01 and $-3.6 \mathrm{dBm}$ for LP11. While at RAU-2 and RAU-1, FEC limit is achieved at $-4.9 \mathrm{dBm}$ for LP01, $-2.8 \mathrm{dBm}$ for LP1 1 and $-2.2 \mathrm{dBm}$ for LP01, $-0.6 \mathrm{dBm}$ for LP11, respectively. The reason behind this particular trend may be understood from Eq. (10) which states that the variance of light intensity fluctuation can be increased by either increasing the length of FSO link or the value of $C_{n}^{2}$. By increasing the value of $C_{n}^{2}$, the variance increases which increases the BER which ultimately degrades the system performance. It may be noted that the results discussed so far are without the mode coupling effects commonly known as intra-model and inter-modal coupling in the fiber. Intramode coupling effect do not contribute significantly due to MGDM, so it can be ignored. However, inter-mode coupling always has a detrimental effect as compared to intra-mode coupling which obviously degrades the signal quality [39]. We observed around $1.2 \mathrm{~dB}$ penalty, after introducing crosstalk while keeping all other simulation parameters same, as shown in Fig. 7 for the worst-case scenario observed at RAU-3 for $C_{n}^{2}=5 \times 10^{-14} \mathrm{~m}^{-2 / 3}$. In a nutshell, FEC limit of BER for both PAM-4 DL transmission is achieved at lower ROP for RAU-1, and for UL transmission, it is achieved at lower ROP for RAU-3. It may be concluded from the results that the wavelengths of multi-wavelength comb can further be exploited by employing MGDM to increase the capacity of the hybrid FSO-MMPOF link. The length of FSO link and MMPOF between each RAU are appropriately selected to achieve BER in acceptable range for each channel.

\section{Conclusion}

A full duplex hybrid FSO-MMPOF architecture using ring topology is proposed for short-range wireless access networks by employing MGDM and wavelength reuse techniques to transmit data between CU and RAUs. A comb of multiple sidebands is generated using a single laser and RF source. LP01 and LP11 modes of each optical sideband are used to provide mm-wave services at each RAU by transmitting PAM-4 signals over hybrid FSO-MMPOF link. Data rate of $2 \times 12 \mathrm{Gbps}$ at $60 \mathrm{GHz}$ is achieved at each RAU for both DL and UL transmissions by employing this cost effective hybrid FSO-MMPOF architecture. Wavelength reuse technique is used to achieve simplicity in terms of mmwave generation by optical HD in RAUs to avoid the use of expensive RF local oscillators for mm-wave generation. The FEC limit, $3.8 \times 10^{-3}$, for PAM-4 signal is achieved for weak turbulence at ROP $-11.5 \mathrm{dBm},-10.8 \mathrm{dBm}$, and

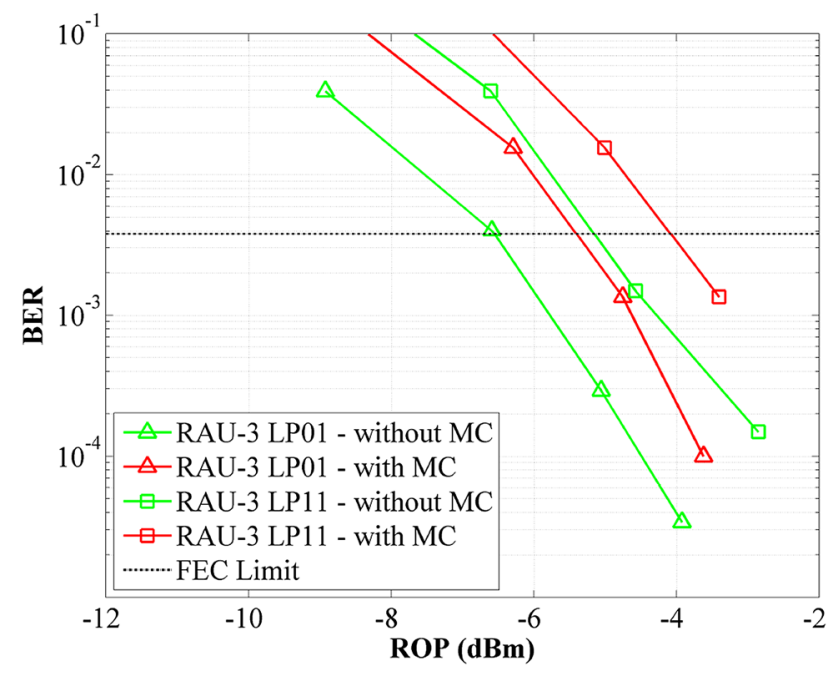

Fig. 7 BER versus received optical power at RAU-3 with and without mode coupling for $C_{n}^{2}=5 \times 10^{-14} \mathrm{~m}^{-2 / 3}$ 
$-9.2 \mathrm{dBm}$ for RAU-1, RAU-2, and RAU-3, respectively. The proposed architecture is capable of providing better coverage and enhancing data rate with better BER performance.

\section{Declarations}

Conflict of interest On behalf of all authors, the corresponding author states that there is no conflict of interest.

Open Access This article is licensed under a Creative Commons Attribution 4.0 International License, which permits use, sharing, adaptation, distribution and reproduction in any medium or format, as long as you give appropriate credit to the original author(s) and the source, provide a link to the Creative Commons licence, and indicate if changes were made. The images or other third party material in this article are included in the article's Creative Commons licence, unless indicated otherwise in a credit line to the material. If material is not included in the article's Creative Commons licence and your intended use is not permitted by statutory regulation or exceeds the permitted use, you will need to obtain permission directly from the copyright holder. To view a copy of this licence, visit http://creativecommons.org/licenses/by/4.0/.

\section{References}

1. Leonberger, F.J., Melchior, H., Osgood, R.M., Yoshikuni, Y.: Introduction to the issue on integrated optics and optoelectronics. IEEE J. Sel. Top. Quantum Electron. 6(1), 1 (2000)

2. Chowdhury, M.Z., Hasan, M.K., Shahjalal, M., Hossan, M.T., Jang, Y.M.: Optical wireless hybrid networks: trends, opportunities, challenges, and research directions. IEEE Commun. Surv. Tutor. 22(2), 930 (2020). https://doi.org/10.1109/COMST.2020. 2966855

3. Goldsmith, A.: Wireless Communications. Cambridge University Press, Cambridge (2005). https://doi.org/10.1017/CBO9780511 841224

4. Thomas, V.A., Ghafoor, S., El-Hajjar, M., Hanzo, L.: The Rap on ROF radio over fiber using radio access point for high data rate wireless personal area networks. IEEE Microw. Mag. 16(9), 64 (2015)

5. Thomas, V.A., El-Hajjar, M., Hanzo, L.: Performance improvement and cost reduction techniques for radio over fiber communications. IEEE Commun. Surv. Tutor. 17(2), 627 (2015)

6. Raza, A., Ghafoor, S., Butt, M.F.U.: MIMO-enabled integrated MGDM-WDM distributed antenna system architecture based on plastic optical fibers for millimeter-wave communication. Photon. Netw. Commun. 35(2), 265 (2018)

7. FTTx roll-out and capex worldwide: forecasts and analysis 20142019. [online] Available: https://www.marketresearch. com/product/sample-8281254.pdf. Accessed 2019

8. Chowdhury, M.Z., Hasan, M.K., Shahjalal, M., Shin, E.B., Jang, Y.M.: Opportunities of optical spectrum for future wireless communications. In: International conference on artificial intelligence in information and communication (ICAIIC), pp. 004-007, (2019)

9. Willebrand, H., Ghuman, B.S.: Free Space Optics: Enabling Optical Connectivity in Today's Networks. SAMS Publishing, United States (2002)

10. Miglani, R., Singh, M.L.: Performance evaluation of free space optical link using mid and far infrared wavelengths in turbulent atmospheric conditions. In 4th International workshop on fiber optics in access network (FOAN), pp. 31-35. DOI: https://doi. org/10.1109/FOAN.2013.6648822,(2013)
11. Liu, Y., Li, H.: Research on transmission performance of multiple-input multiple-output optical communication in atmospheric turbulence. In: 2020 12th International conference on measuring technology and mechatronics automation (ICMTMA), pp. 630-633, (2020)

12. Li, M.: MMF for high data rate and short length applications. In: Optical fiber communication conference (OFC), pp. 1-3, (2014)

13. Iqbal, S., Iqbal, M., Raza, A., Mirza, J., Ghafoor, S., El-Hajjar, M., Butt, M.F.U.: Linearly polarized modes enabled PAM-4 data transmission over few-mode fiber for data center interconnect. Electron. Lett. 56(21), 1125 (2020)

14. Al-Musawi, H.K., Cseh, T., Abadi, M.M., Ng, W.P., Ghassemlooy, Z., Udvary, E., Berceli, T.: Experimental demonstration of transmitting LTE over FSO for in-building POF networks. In: 17th International conference on transparent optical networks (ICTON), pp. 1-4, (2015)

15. Yeh, C.H., Gu, C.S., Guo, B.S., Chang, Y.J., Chow, C.W., Tseng, M.C., Chen, R.B.: Hybrid free space optical communication system and passive optical network with high splitting ratio for broadcasting data traffic. J. Opt. 20, 125702 (2018)

16. Huang, X.H., Lu, H.H., Donati, S., Li, C.Y., Wang, Y.C., Jheng, Y.B., Chang, J.C.: Two-way wireless-over-fibre and FSO-overfibre communication systems with an optical carrier transmission. Laser Phys. 28, 076207 (2018)

17. Li, C., Lu, H., Tsai, W., Huang, X., Wang, Y., Chen, Y., Wu, Y.: A flexible two-way PM-based fiber-FSO convergence system. IEEE Photon. J. 10(2), 1 (2018)

18. Iqbal, S., Raza, A., Butt, M.F.U., Ghafoor, S., El-Hajjar, M. : A full-duplex radio over fiber architecture employing $12 \mathrm{Gbps} 16$ $\times 16$ optical multiple input multiple output for next-generation communication networks. Trans. Emerg. Telecommun. Technol. https://doi.org/10.1002/ett.3910

19. Chatti, I., Baklouti, F., Chekir, F., Attia, R.: Comparative analysis of MIMO-based FSO and MIMO-based MGDM communications. Opt. Rev. 26, 631 (2019)

20. Beas, J., Castanon, G., Aldaya, I., Aragon-Zavala, A., Campuzano, G.: Millimeter-wave frequency radio over fiber systems: a survey. IEEE Commun. Surv.Tutor. 15(4), 1593 (2013)

21. Zhong, K., Zhou, X., Gui, T., Tao, L., Gao, Y., Chen, W., Man, J., Zeng, L., Lau, A.P.T., Lu, C.: Experimental study of PAM-4, CAP-16, and DMT for $100 \mathrm{~Gb} / \mathrm{s}$ short reach optical transmission systems. Opt. Express 23(2), 1176 (2015)

22. IEEE P802.3bs $400 \mathrm{~Gb} / \mathrm{s}$ ethernet task force. [online] Available: http://www.ieee802.org/3/bs/. Accessed date 2019

23. Raza, A., Zhong, K., Ghafoor, S., Iqbal, S., Adeel, M., Habib, S., Butt, M.F.U., Lu, C.: SER estimation method for 56 GBaud PAM-4 transmission system. Chin. Opt. Lett. 16(4): 040604 (2018). http://col.osa.org/abstract.cfm?URI=col-16-4-040604

24. Mirza, J., Ghafoor, S., Hussain, A.: A full duplex ultrawideband over free-space optics architecture based on polarization multiplexing and wavelength reuse. Microw. Opt. Technol. Lett. 62, 3999-4006 (2020)

25. Hmood, J.K., Emami, S.D., Noordin, K.A., Ahmad, H., Harun, S.W., Shalaby, H.M.H.: Optical frequency comb generation based on chirping of Mach-Zehnder Modulators. Opt. Commun. 344, 139 (2015). https://doi.org/10.1109/LPT.2005.853035

26. Zhang, L., Song, Y., Zou, S., Li, Y., Ye, J., Lin, R.: Flat frequency comb generation based on Mach-Zehnder modulator and phase modulator. In: IEEE 12th International conference on communication technology, pp. 211-213, (2010)

27. Koyama, F., Iga, K.: Frequency chirping in external modulators. J. Lightwave Technol. 6(1), 87 (1988)

28. Ho, K.P., Kahn, J.M.: Spectrum of externally modulated optical signals. J. Lightwave Technol. 22(2), 658 (2004)

29. Korenev, B.G.: Bessel Functions and Their Applications. CRC Press, United States (2002) 
30. Mirza, J., Ghafoor, S., Hussain, A.: All-optical generation and transmission of multiple ultrawideband signals over free space optical link. Opt. Eng. 58(5), 056103 (2019)

31. Andrews, L.C., Phillips, R.L.: Laser Beam Propagation Through Random Media. SPIE Press, United States (2005)

32. Lee, E.J., Chan, V.W.: Part 1: optical communication over the clear turbulent atmospheric channel using diversity. IEEE J. Sel. Areas Commun. 22(9), 1896 (2004)

33. Davaslog lu, K., Cagiral, E., Koca M. : Free space optical ultrawideband communications over atmospheric turbulence channels. Opt. Exp. 18(16), 16618 (2010)

34. Hanzawa, N., Saitoh, K., Sakamoto, T., Matsui, T., Tomita, S., Koshiba, M.: Demonstration of mode-division multiplexing transmission over $10 \mathrm{~km}$ two-mode fiber with mode coupler. In: Optical fiber communication conference and exposition and the national fiber optic engineers conference, pp. 1-3, (2011)

35. Nanzer, J.A., Callahan, P.T., Dennis, M.L., Clark, T.R., Jr.: Photonic signal generation for millimeter-wave communications. Johns. Hopkins APL Tech. Dig. 30(4), 299 (2012)
36. Chagnon, M., Osman, M., Poulin, M., Latrasse, C., Gagné, J.F., Painchaud, Y., Paquet, S., Lessard, C., Plant, D.: Experimental study of $112 \mathrm{~Gb} / \mathrm{s}$ short reach transmission employing PAM formats and $\mathrm{SiP}$ intensity modulator at $1.3 \mu \mathrm{m}$. Opt. Express 22(17), 21018 (2014)

37. Li, M., Chen, X., Nolan, D.A.: Ultra low PMD fibers by fiber spinning. in Optical Fiber Communication Conference, 2004. OFC 2004, Vol. 2, p. 3. (2004)

38. Bloembergen, N.: Nonlinear optics: past, present, and future. IEEE J. Sel. Top. Quantum Electron. 6(6), 876 (2000)

39. Awad, M., Dayoub, I., Okassa M'Foubat, A., Rouvaen, J.M.: The inter-modes mixing effects in mode group diversity multiplexing. Opt. Commun. 282(19), 3908 (2009)

Publisher's Note Springer Nature remains neutral with regard to jurisdictional claims in published maps and institutional affiliations. 